\title{
A Lumped Electro-Thermal Model for Li-Ion Cells in Electric Vehicle Application
}

\author{
Kamyar Makinejad ${ }^{1}$, Raghavendra Arunachala $^{1}$, Sabine Arnold ${ }^{1}$, Hassen Ennifar $^{1}$, Han Zhou $^{1}$, \\ Andreas Jossen ${ }^{2}$, Wen Changyun ${ }^{3}$ \\ ${ }^{1}$ TUM CREATE, Singapore, kamyar.makinejad@tum-create.edu.sg \\ ${ }^{2}$ Technische Universität München, (TUM), Munich, Germany; Institute for Electrical Energy Storage Systems (EES) \\ 3Nanyang Technological University (NTU), school of electrical \& electronic engineering
}

\begin{abstract}
A lumped Electro-thermal model of a large format high power Li-Ion cell is introduced in this paper. The model is able to meet the real time implementation requirements; hence it finds its application in Battery Management System (BMS) of an Electric Vehicle (EV).

The model is evaluated in Hardware in the Loop (HIL) setup to verify online estimation of cell surface and internal temperature estimation for an on-board EV application.

In this study, the cell is considered as a single homogeneous layer and the heat is generated in the centre point of the cell and flows in one direction towards the surface. For this modelling purpose, reversible and irreversible heat in the cell is considered. Irreversible heat consists of the Joule heating effect due to internal resistance of the cell, for instance these values are then calculated with sufficient electrical cell model and evaluated both offline and in real time calculation. Reversible heat is a result of entropy effect which can be negative or a positive value depending on the direction of current flow during charging and discharging process of the cell. Other heat transfer mechanism such as conductive heat transfer and convective heat transfer are also included into the model.

This paper introduces a reference case test used to calculate the required necessary coefficients both for parameterization of electrical model and thermal model. The battery setup in the laboratory for measuring the cell surface temperature as reference data as well as cell sandwich setup for evaluating the internal temperature of the cells is explained in detail. Fundamental equations to develop the thermal model are introduced and the model is evaluated in both offline and real time mode.
\end{abstract}

Keywords: Li-Ion cell, Equivalent circuit model, Thermal model, Battery management system, Hardware in the loop

\section{Introduction}

In EVs, the battery is the main energy provider which is responsible for the supply of all the electrical components. These components differ from each other in their energy requirements, thus a high dynamic range of energy is needed. This dynamic aspect becomes critical for high voltage implementation. When several cells are connected together in series and parallel, this forms the battery pack of an EV. Therefore, a reliable and accurate BMS is required to afford the adequate amount of power and energy for various applications in an EV. This leads to the necessity of keeping the batteries in optimal conditions, decreasing their losses and protecting them from potential damage such as overheating by high currents, overcharging and over discharging.

Since providing these optimal working conditions for the cells and gaining critical information about the cell real-time and future status such as amount of available energy, remaining life time and many other valuable information can't be realized by direct measurement via physical sensors, developing models such as Equivalent Circuit Model (ECM), thermal model, ageing model and various algorithms for estimation of State of 
Charge (SOC), State of Health (SOH), State of Function (SOF) and many others as the heart of the BMS of an EV is necessary.

Figure 1 demonstrates the interaction between these models and algorithms in the form of a flowchart. Some parts of this flowchart are described more in detail in this work but not all as they were outside the scope of this paper.

In section 2, the experimental setup is explained and the required test data such as Voltage (V), Current (I) and Temperature (T) are logged via high speed data logger.

Section 3 describes the cell parameterization and cell voltage model, which is also known as ECM. SOC model is not explained in this paper but in the simulation it was running in parallel to the EMC and cell thermal model to generate the required parameter for the cell simulation as the parameters of the cell are all SOC dependant too. In section 4, cell thermal model is demonstrated and the interaction with the EMC is explained.

The outcome of the cell EMC, thermal model, SOC model, SOH model and eventually SOF model are up-scaled to create the overall battery pack model. The battery pack model is implementable on the target microcontroller or the BMS system.

Parameter adjustment shown in the flowchart is due to the fact that cell parameter will change over time for instance by ageing or other external conditions such as temperature variation, hence the new parameter should be considered for both ECM and thermal model.

By using cell ECM [1] and [2], one can easily realize that in general any successful and functional BMS should take advantage of this model, in addition to thermal model, SOC estimator and $\mathrm{SOH}$ estimator etc. Since SIMULINK is suitable for offline data analysis, DS2202 HIL I/O board system from Dspace was used in this work, which enables us to read physical signals such as voltages, temperature and current in real time with the help of Analog to Digital Converter (ADC). These signals can be used in the compiled SIMULINK model in the ControlDesk. ControlDesk is a dSPACE experiment software for single working environment to run tasks such as HIL, Rapid control prototyping (RCP) etc. Then the cell voltage, temperature, $\mathrm{SOC}, \mathrm{SOH}$ and other models can be simulated in real time for evaluation based on the desired test plan written for the battery cycler.

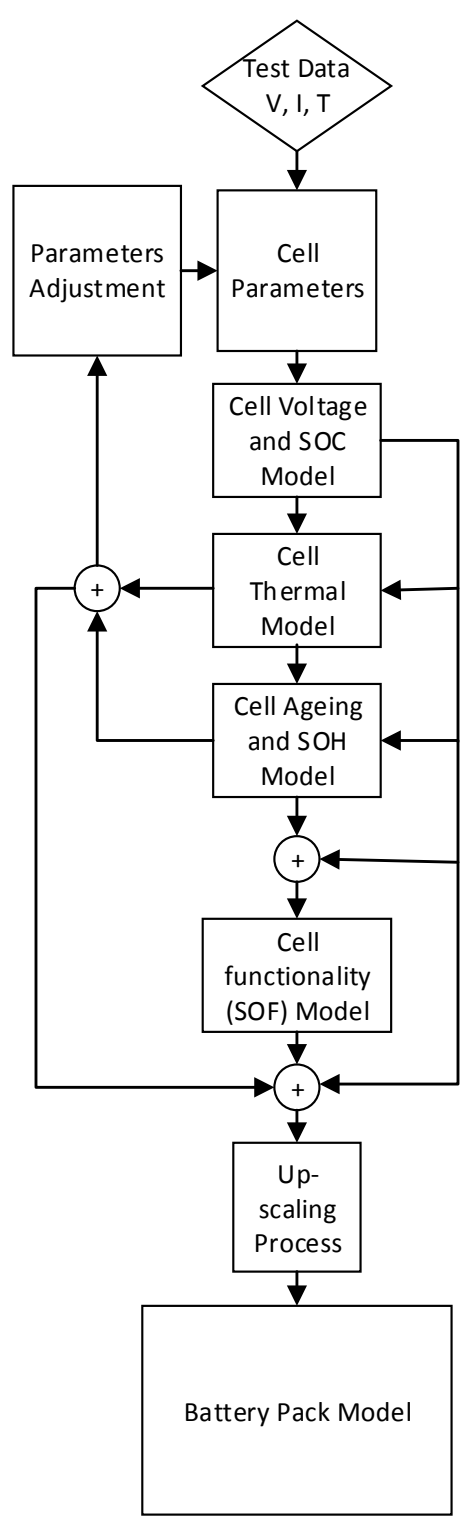

Figure 1: Flowchart of the investigation on the Li-Ion cell

\section{Experimental}

\subsection{Test Setup}

High power Li-Ion pouch cell was used in this experiment. The cathode material is nickel manganese cobalt oxide (NMC) and the anode material is graphite, found by X-Ray Diffraction (XRD) analysis. The electrolyte is a solvent of EC:EMC and LiPF6 salt which is also studied by Botte et al. in terms of thermal stability by means of Differential Scanning Calorimeter (DSC) [3]. 
Three large format Li-ion pouch cells as shown in Figure 2 with the nominal capacity of $63 \mathrm{Ah}$ and nominal voltage of $3.7 \mathrm{~V}$ were used in the experiment. Figure 3 shows the test bench. High current battery cycler from Digatron industries with current ratings up to $300 \mathrm{~A}$ with additional parallel option of 4 circuits to reach $1200 \mathrm{~A}$, a $256 \mathrm{~L}$ Memmert temperature chamber with the operating range between $-40{ }^{\circ} \mathrm{C}$ to +120 ${ }^{\circ} \mathrm{C}$, host computer and other equipment such as data loggers and precision current sensors were the part of this test bench setup. To evaluate the effectiveness of the algorithms, both new and aged cells cycled based on the flowchart shown in Figure 4 were considered. RTP in this flowchart stands for Reference Performance Test. RTP is the first step in the design of the test for cell parameterization, which includes performing Hybrid Pulse Power Characterization (HPPC) test [4] as shown in figure 5. This test was repeated every 100 cycles under various conditions for instance different temperature level as shown in Figure 4. To have statistically reliable data, three cells were used for each test matrix and the parameterization result was the averaged value among these cells under each condition. By analysing separately the charge and discharge pulses, cell parameters were calculated.

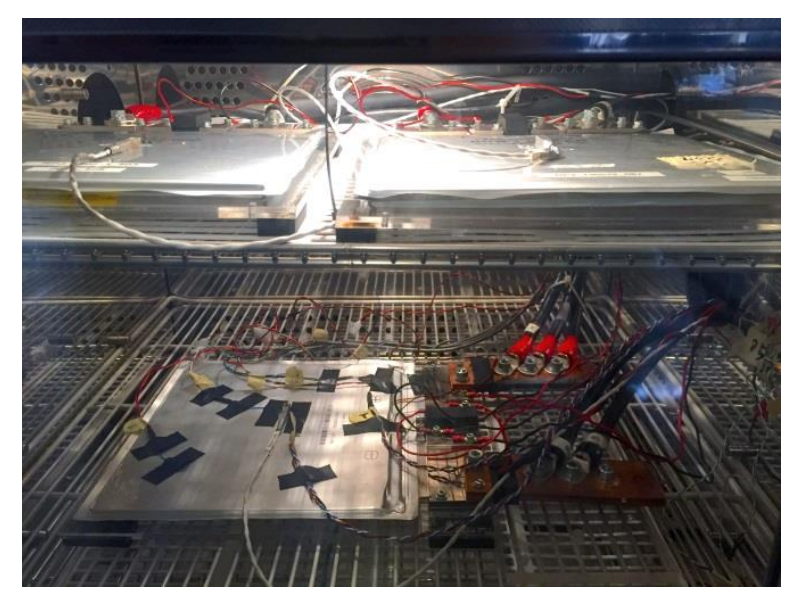

Figure 2: 3 Li-Ion cells under test

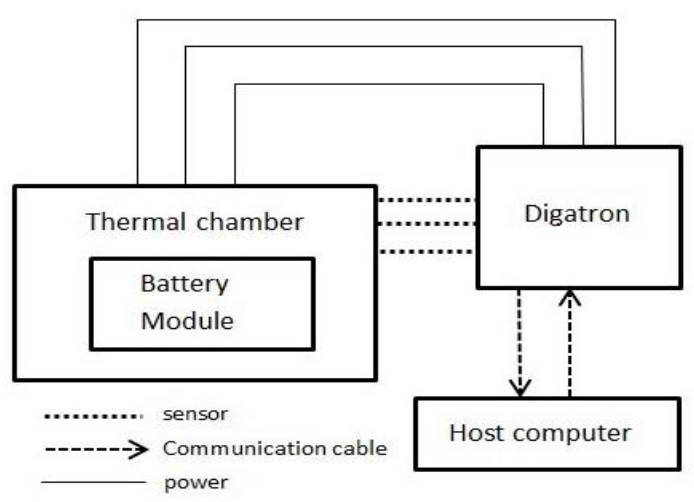

Figure 3: Test bench

Figure 4 demonstrates the procedure of cycling ageing tests, which includes required characterization test to parameterize the cell model presented in figure 6 .

Based on this flowchart, 3 new cells underwent RTP test, which is a combination of Electrochemical Impedance Spectroscopy (EIS), discharge capacity test and HPPC test. The EIS results of fresh and aged cells are not presented in this paper as the aim of this research is to develop time domain model of the cell. The HPPC test was done for each cell at different test conditions, with the aim of calculating the parameters of the cell. Section 3 explains the application of HPPC test to develop the parameters of the ECM model.

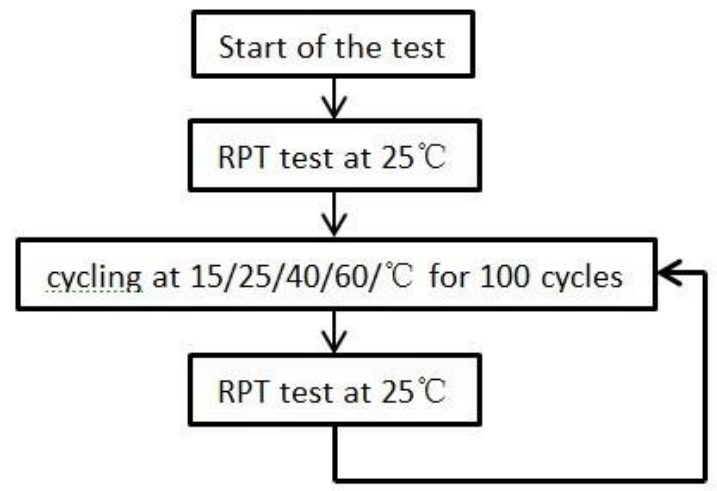

Figure 4: Ageing test flowchart used in this case study 


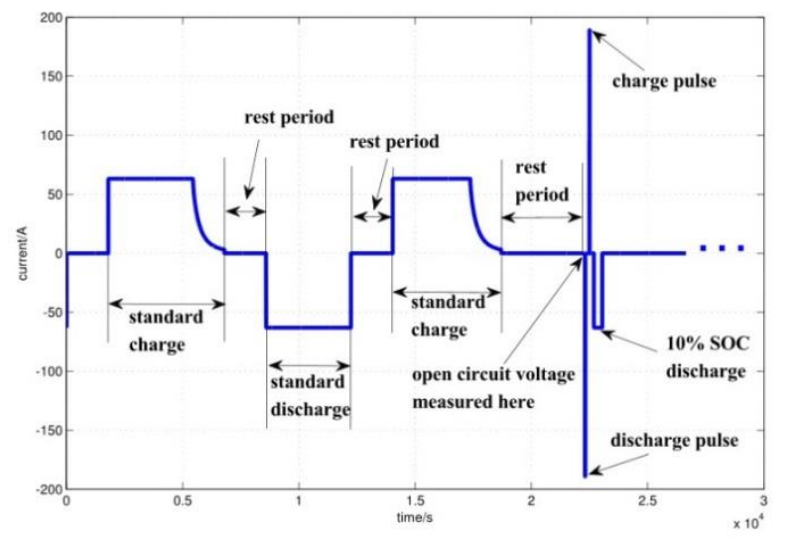

Figure 5: HPPC test to characterize the cell

The cycling ageing test will continue until the time which the cell discharge capacity reaches $80 \%$ of the initial discharge capacity at the time the cell was new. By definition when the cell discharge capacity is reduced by $20 \%$ the cell is considered to have reached its End of Life (EOL) for EV application.

Other method such as cell impedance monitoring is also being used to indicate if the cell is at EOL or is not able to deliver the required power for the specific application.

\section{Cell Equivalent Circuit Model (ECM)}

ECM is an electrical approach for representation of the internal/external behaviour of a cell. Other approaches are available for representation of the electrochemical internal/external behaviour of the cell which is described in section 5. However the focus of this paper is mostly on the methods which are applicable to target low cost microcontrollers or the BMS of an EV [5]. It is noteworthy to mention that this high power LiIon cell is being used to power TUM CREATE's electric taxi called EVA which is developed for tropical weather in Singapore.

In this section, parameterization method of a LiIon cell is explained.

\subsection{Cell Parameterization}

In figure 6 , the required cell ECM to provide inputs for cell thermal model to estimate internal/external temperature is presented.

Analysis shows that this model is sufficient for accurate voltage simulation of the cell without the help of special controller or estimators such as Kalman Filter (KF) family. However it is noteworthy to mention that all the calculated parameters of the ECM model are dependent on SOC, Temperature and ageing. The test matrix for the ageing test is extensively explained in [6].

Two conventional methods to determine any energy storage system parameters are either in frequency domain such as EIS with sinusoidal excitation or with triangular excitation as discussed in [7] or in time domain such as Hybrid Pulse Power Characterization (HPPC) test. HPPC current profile is presented in figure 5 and used to parameterize the high power Li-Ion pouch cell.

Cell parameterization can be performed both offline or online [5]. In our study, cell initial parameters were calculated in offline mode and later these parameters were updated by minimizing the mean square error between the measured voltage and the simulated voltage. We refer to simulated voltage as $V_{\text {out }}$ shown in figure 6 .

Other parameter in this figure, is $V_{O C V}(S O C, T, n)$ representing the open circuit voltage of the cell which is a function of SOC, temperature (T) and cycle number (n) and can be measured after sufficient rest time under no load $(I=0)$. To obtain the parameters of the cells, experiments were conducted at different temperature conditions and cycling aging performed by several charging and discharging cycles. In order to consider the temperature effect on the cell parameters, ageing tests were conducted at four different temperatures $\left(15{ }^{\circ} \mathrm{C}, 25{ }^{\circ} \mathrm{C}, 40{ }^{\circ} \mathrm{C}\right.$ and $\left.60{ }^{\circ} \mathrm{C}\right)$ and all parameters were calculated based on these temperatures and at different SOC levels and ageing conditions. Parameter values at temperatures other than the mentioned controlled test temperatures were calculated by interpolation of the data. $R_{i}$ represents the series Ohmic resistance of the cell but good caution must be taken into account while connecting the cell in the experimental setup, since any loose or bad wire connection to the cell terminals will introduce additional contact resistance and the results will be different than the reality.

Charge transfer resistance and Solid Electrolyte Interface (SEI) resistance were considered both as $R_{1}$. SEI forms when the cell is charged for the first time, as the result of the reaction of Lithium with the graphite, this process is irreversible and cell loses some of its initial capacity. SEI continues to grow over further charge and discharge cycles of the cell until a steady state thickness is established. Further growth of this layer greatly increases the resistance of the cell. This resistance together with 
$C_{1}$ known as double layer capacitance the time constant $\tau_{1}$ is introduced $\left(\tau_{1}=R_{1} * C_{1}\right)$.

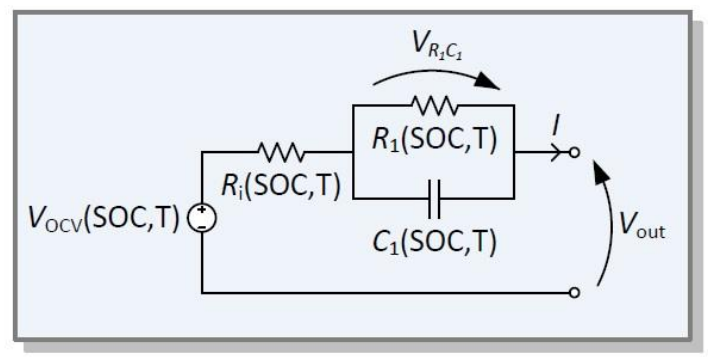

Figure 6: Equivalent circuit model used in simulation

\subsubsection{Calculation of $V_{O C V}(S O C, T)$ for the Model}

By using the test profile shown in figure 5, the $\mathrm{OCV}$ values were measured by giving sufficient rest time to the cell after each charge/discharge pulses.

The OCV-SOC characteristic of the Li-Ion cell is nonlinear. This nonlinearity is shown in Figure 7. In this figure OCP stands for Open Circuit Potential, which means the relaxed no load voltage of the terminals of the cell and considered similar to OCV. It should be noted that enough relaxation time should be given to the cell to attain voltage and temperature equilibrium. Temperature also has effect on OCV values, therefore cells were kept in a controlled environment inside the temperature chambers. Calculating OCV at different SOC values were done by measuring the discharge capacity of the cell and stepwise charging or discharging, based on the specific fractures of the total capacity of the cell.

We considered capturing the OCV values at each $10 \%$ steps in SOC as the cell has steep OCVSOC curve as described in detail in [4].

OCV values are also dependent on the temperature that the cell was cycled at. Figure 8 shows the OCV of the cell cycled at $25{ }^{\circ} \mathrm{C}$ after from 0 cycle (new) to 1400 cycles. Results show that the OCV values of the cell are decreasing over ageing at same cycle ageing condition (e.g. same temperature and test profile), similar results repeats for the other cycling ageing condition at different temperature values. Ageing results are not presented in this paper.
Subsection 3.1.2 analyses the ageing and temperature effect on the internal resistance of the cell.

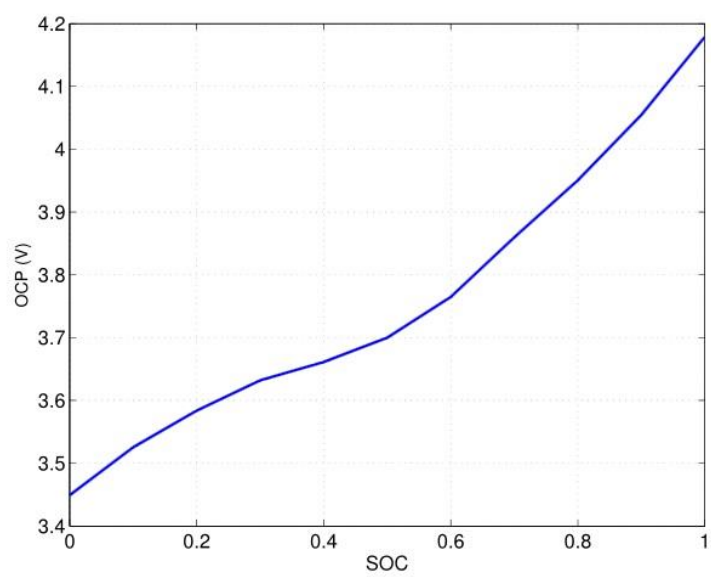

Figure 7: Fresh cell OCV vs SOC at $25^{\circ} \mathrm{C}$

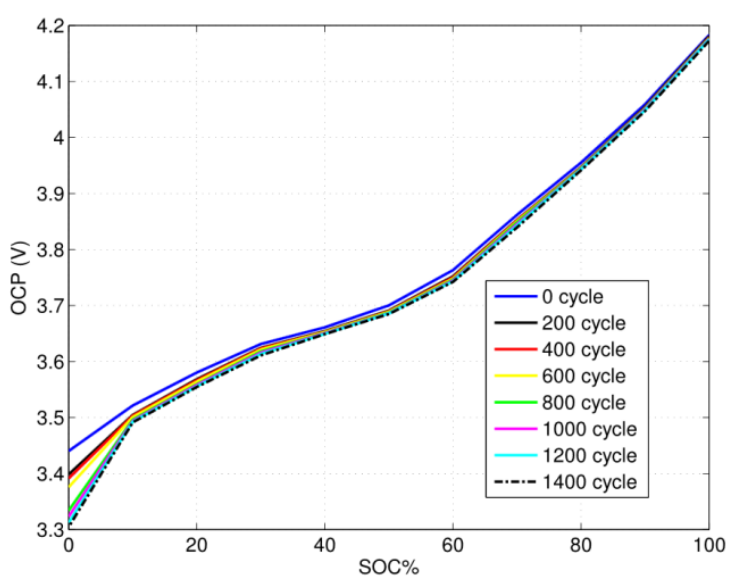

Figure 8: Averaged OCV of 3 cells cycled at $25{ }^{\circ} \mathrm{C}$

\subsubsection{Measurement of Discharge Capacity}

The discharge capacity of the cells cycled at $25{ }^{\circ} \mathrm{C}$ and $40{ }^{\circ} \mathrm{C}$ was measured at every 100 cycles up to 1400 cycles and for the cells cycled at $15^{\circ} \mathrm{C}$ and $60{ }^{\circ} \mathrm{C}$ up to 500 cycles. Cells cycled at $15^{\circ} \mathrm{C}$ and $60{ }^{\circ} \mathrm{C}$ lost more than $20 \%$ of their initial discharge capacity after 600 cycles, therefore the tests had to be terminated for these cells. Looking into the discharge capacity plot, it is revealed that at controlled temperature of $25{ }^{\circ} \mathrm{C}$, the irreversible capacity loss is 4.38 Ah by calculation. These data later are being used to create reference data for SOC estimation and also as reference data to 
evaluate State of Health (SOH) of the cell for comparison purposes with the simulation result.

$\mathrm{SOH}$ of a battery is a measure that describes how much the battery has degraded in health over the course of its lifetime, and is often evaluated by the battery's internal resistance or its ability to deliver a given amount of charge or defined by capacity loss [8] .

By definition SOC represents the amount of the charge currently stored in the cell. Equation 1 shows how cell SOC is related to $\mathrm{SOH}$.

$S O C=Q a / Q n$

$Q_{a}$ denotes the available charge of the cell, and $Q_{n}$ is the total charge of the cell. $Q_{n}$ is subject to the cell ageing and describes the relation between cell SOC and $\mathrm{SOH}$. To realize the above formula as current is the rate of flow of charge and by renaming the charge to cell capacity we have:

$$
\operatorname{SOC}(t)=\frac{\int I(t) d t}{C n}+\operatorname{SOC}_{0}
$$

However equation (2) or coulomb counting method is strongly dependent on current measurement accuracy plus accurate knowledge of the initial cell SOC. A critical SOC definition is, Li-Ion deintercalation/intercalation from anode/cathode material and placing in the cites of the cathode/anode material during discharge/charge, this process is considered as surface SOC. Figure 9 shows the normalized discharge capacity of the cell measured at $1 \mathrm{C}$ rate discharge at $25{ }^{\circ} \mathrm{C}$, as it can be understood from this discharge capacity test that cells cycled at 25 ${ }^{\circ} \mathrm{C}$ and $40{ }^{\circ} \mathrm{C}$ lost only $10 \%$ of their initial discharge capacity after 1400 cycles but the cells cycled at $60{ }^{\circ} \mathrm{C}$ lost $20 \%$ of their initial discharge capacity after about 400 cycles.

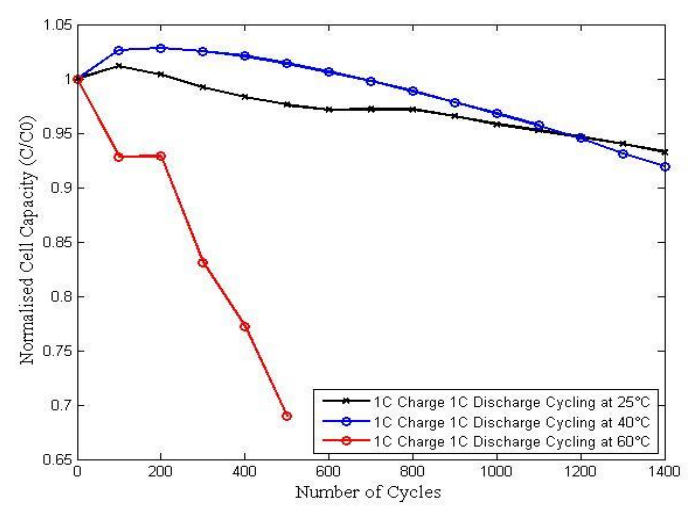

Figure 9: Normalized cell capacity measured at 1C rate discharge at $25^{\circ} \mathrm{C}$

\subsubsection{Calculation of $R_{i}(S O C, T)$}

The cell parameterization algorithm automatically calculates all necessary parameters over different temperature range and cycles of several cells to create reference data, and enable us to study the ageing mechanism of the cell. Cell voltage simulation also depends on how accurate the cell parameters are captured and how robust the model works. Figure 10 shows averaged internal resistance evolution of 3 cells cycled from 0 to 1400 cycles (standard procedure charge and discharge based on manufacturer standards) at $25^{\circ} \mathrm{C}$, in this figure, $R_{i}$ is plotted every 200 cycles. Similar data at different temperatures and at different cycle numbers were collected and analysed but are not explained in this paper. It is noteworthy to mention that the internal resistance is mainly the sum of unwanted wirings resistance, bulk metal resistance in the current collectors and terminals, active material resistance and electrolyte resistance.

$R_{i}$ values were calculated from the HPPC pulse data, this pulses are either charge or discharge current of at least $600 \mathrm{~A}$.

We consider the instantaneous change in the relaxed voltage value as the series resistance value, hence fast data logger is necessary to calculate the correct value of this series resistance. It is obvious that the series resistance increases due to ageing, which results in reduction of power capability of the cell and more internal heat generation. The heat generation due to increase in series resistance is explained in section 4 .

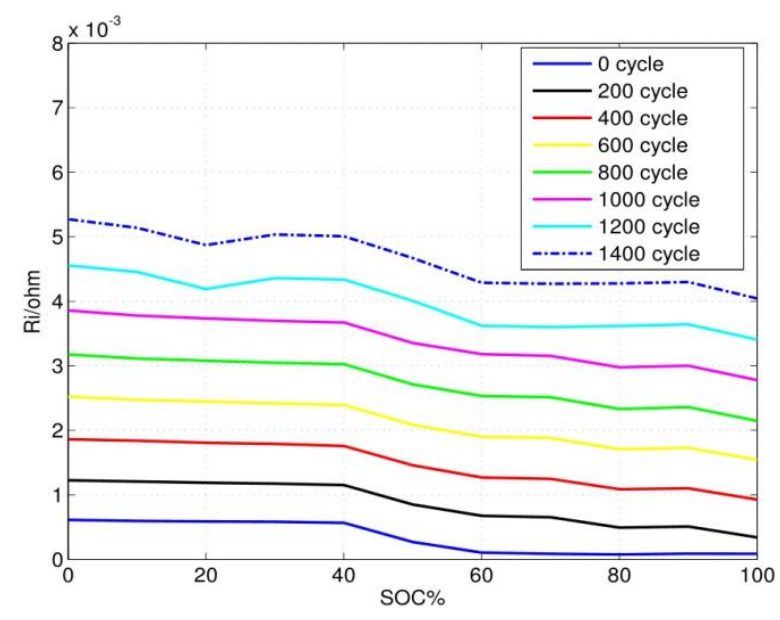

Figure 10: Averaged internal resistance evolution of 3 cells cycled at $25^{\circ} \mathrm{C}$ 


\subsubsection{Calculation of $R_{1}(S O C, T)$ and $C_{1}(S O C, T)$}

Figure 11 shows the variation of series resistance $R_{i}$ at different SOC with respect to 0,200 and 400 cycles under four different temperatures (15 ${ }^{\circ} \mathrm{C}, 25{ }^{\circ} \mathrm{C}, 40{ }^{\circ} \mathrm{C}$ and $\left.60{ }^{\circ} \mathrm{C}\right)$. For fresh cells $(0$ cycle) there are small differences in $R_{i}$ values at different temperatures. However for cells cycled at $60^{\circ} \mathrm{C}$ had a significant increase of series resistance, compared to cells cycled at other temperatures. Figure 12 and 13 show the parameterized value of $R_{1}(S O C, T, n)$ and $C_{1}(S O C, T, n)$. Similar to OCV, they are SOC, temperature and cycle number dependant.

In figures 11,12 and 13 , temperature is represented with different colours in each plane.

Each of these parameters are plotted in the planes

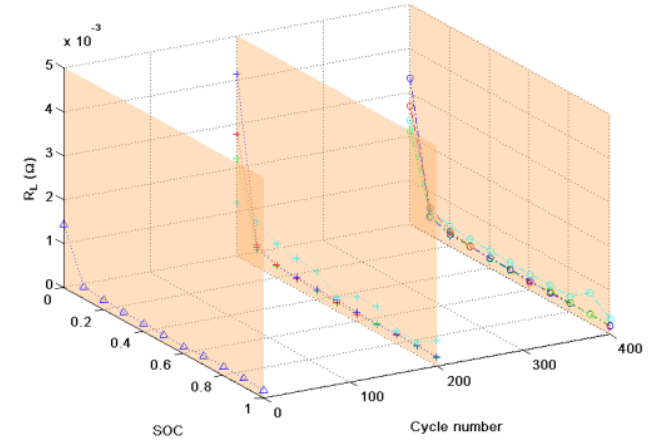

Figure 11: $R_{i}$ as a function of SOC, cycle number and $\mathrm{T}$

with certain cycle number and certain temperatures $(\mathrm{T})$ to clearly demonstrate the change of the parameters over different SOC values, Temperature and cycle number.

Each test matrix includes 3 similar cells tested at 4 different temperatures $\left(15{ }^{\circ} \mathrm{C}, 25^{\circ} \mathrm{C}, 40{ }^{\circ} \mathrm{C}\right.$ and $\left.60{ }^{\circ} \mathrm{C}\right)$. These results are the averaged parameter values among the 3 cells at the same test conditions.

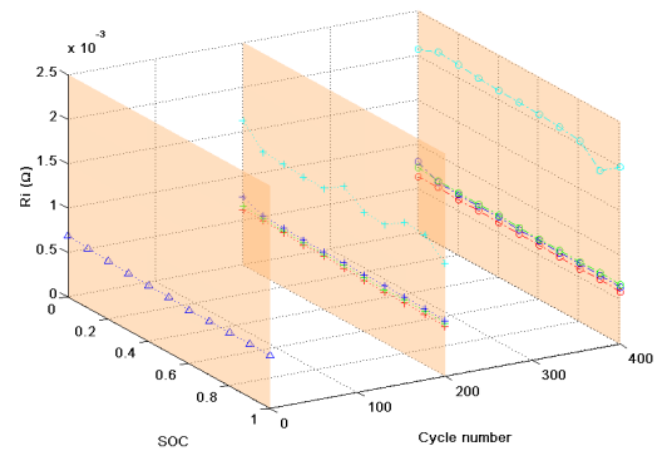

Figure 12: $R_{1}$ as a function of SOC, cycle number and $\mathrm{T}$

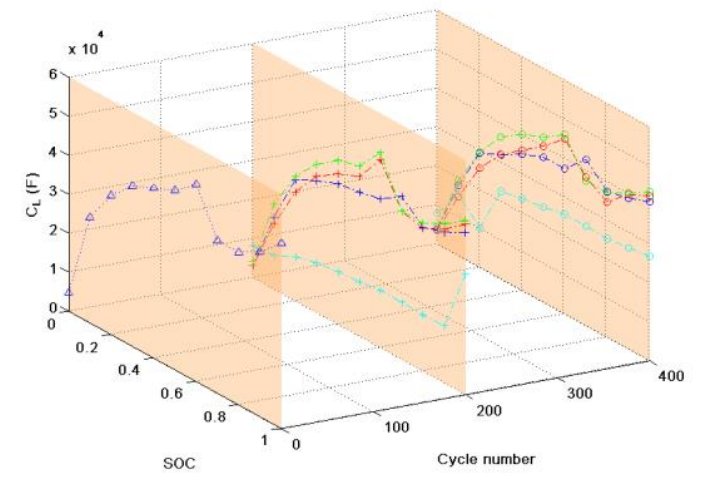

Figure 13: $\mathrm{C}_{1}$ as a function of SOC, cycle number and T

\subsection{Simulation of ECM Model}

We described parameterization and parameters of the ECM model in subsection 3.1. This part will demonstrate the voltage simulation of the model suggested in figure 6 , however it is possible to estimate $\mathrm{SOC}, \mathrm{SOH}$ and $\mathrm{SOF}$ with the gathered data, parameters and the proposed cell model, but this task is outside the scope of this paper and we only deal with the cell thermal model in section 4 and will show how a cell thermal model is developed over the basis of cell ECM model and is able to estimate both cell surface and internal temperature.

The ECM model also considered ageing of the cell and the parameters were calculated over few hundred cycles.

Ageing mechanism is a complex process occurring due to structural and chemical changes in the components of cell or the material used in the cell. It is characterised by deterioration of battery performance with time and usage. Cell ageing can be classified into calendar ageing and cycling ageing. In the present work we focus mainly on the cycle life ageing of the cells. Most literatures associate ageing mechanism with active material degradation, formation and growth of solid electrolyte interface (SEI) layers and electrolyte decomposition. However very recently, studies show that ageing mechanism can be linked to separator degradation [8], chemistry, cell form and geometry, which cause non-uniform utilisation of active material and results in inhomogeneous ageing. In the present frame work we have only discussed the electrochemical part of cell ageing. In tropical megacities like Singapore, the ambient temperature is quite high and the battery pack should be equipped with better cooling units. 
Literature works show that high temperature has severe effect on cell lifetime with different chemistry due to accelerated ageing [9]. Therefore the main function of the cooling units should be to keep the battery pack temperature at an optimal level and improve its performance and lifetime.

To simulate the terminal voltage of the cell based on Figure 6:

$V_{\text {out }}=V_{O C V}(S O C, T, n)+V_{R 1, C 1}(S O C, T, n)+\ldots$ $R_{i}(S O C, T, n) * I$

In figure 14 , the comparison of the simulation and measured voltage response shown is figure 16 (b) is demonstrated. The simulation is based on equation 3. This simulation model took advantage of model parameters and ECM as discussed in previous section. It is noteworthy to know that the ECM simulation model doesn't take advantage of any corrective algorithms and methods such as Kalman Filter (KF) family at this stage. As mentioned, estimation of cell hidden parameters such as SOC, SOF and $\mathrm{SOH}$ is out of the scope of this paper and we only demonstrate modelling technique for terminal voltage and temperature estimation.

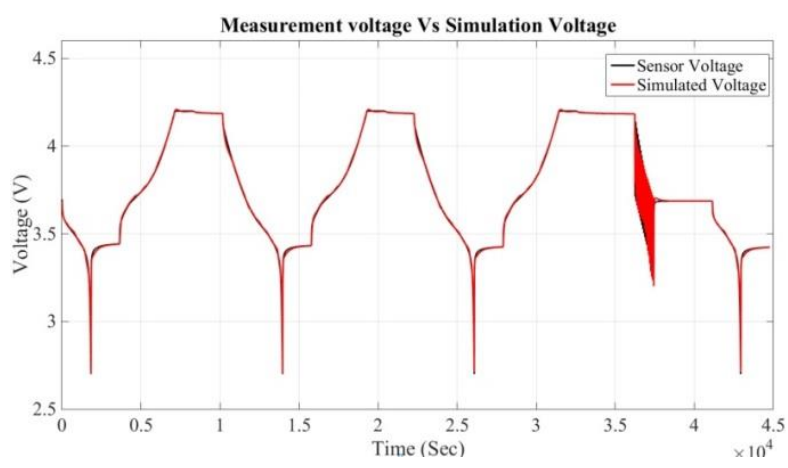

Figure 14: Comparison of measurement and simulation for input current profile shown in figure 16 (b)

\section{Cell Thermal Model}

The objective of this work is to simulate the internal and external (by making cell sandwich) cell temperature and evaluate the simulation results with measured values by Dspace Hardware in the Loop (HIL) real time simulation as shown in figure 15 .
This section introduces a lumped thermal model that simulates both surface and internal temperature.

Similar to section 2, where a HPPC test profile was used to parameterize the cell to develop the ECM, a specific test profile was developed and implemented on the same cell to derive the parameters of the cell thermal model, see figure 16 (a). To collect reliable temperature data, several accurate and sensitive temperature sensors as shown in figure 2, were attached to the cell surface. The experimental setup is similar to the setup shown in figure 2 and 3.

As shown in figure 18 , there is a considerable temperature rise on the surface of the cell, however the ambient temperature value doesn't change much (shown in red colour) as the cell is placed inside a temperature chamber and the ambient temperature is set to $25^{\circ} \mathrm{C}$.

The following subsection demonstrates heat generation mechanism, test profile to get the parameters and the method to model this temperature rise and any other temperature variation on the cell surface as the result of different current profile inputs.

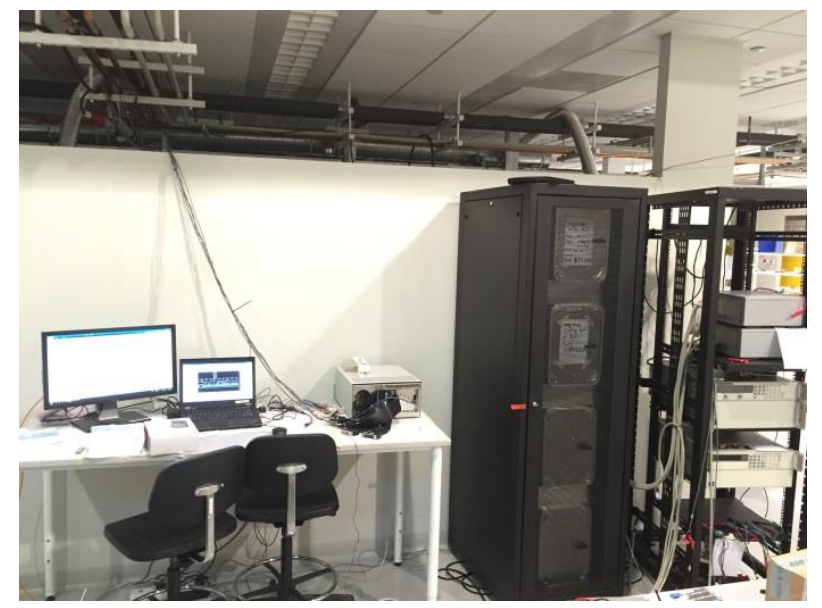

Figure 15: Test setup for HIL implementation for real time implementation of cell models and algorithms

\subsection{One Dimensional Thermal Analysis Work and Heat Generation Mechanism}

This part describes the heat Generation and thermal analysis of the Li-polymer $63 \mathrm{Ah}$ pouch 
cell with the aid of two different softwares: MATLAB SIMULINK and COMSOL which is explained in section 5 .

To start, we briefly speak about different heat mechanisms in Li-Ion cell and the dominating ones responsible for the temperature rise of the cell.

Different heat transfer mechanisms to be considered to simulate the temperature model of the cell are as follows:

- Heat conduction

- Heat convection

- Heat Radiation

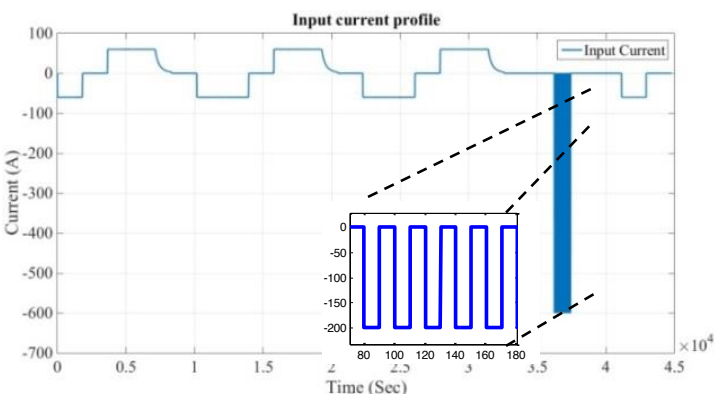

Figure 16 (a)

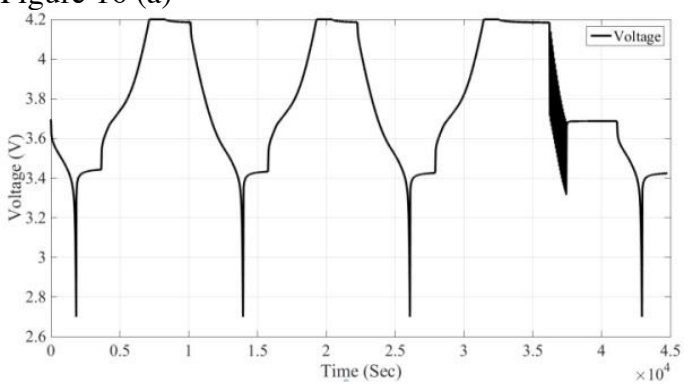

Figure 16 (b)

Figure 16: Experimental test on the pouch cell required to get the initial parameter for cell thermal mode. (a) current profile with 600 A consecutive pulses (10 second pulse), (b) voltage response to the input current profile

Temperature levels for Li-Ion cell environment in EV's and HEV's are assumed to be in between and, most of the radiation heat is absorbed by the cell itself because it is composed from solid materials with high heat capacity. Moreover the temperature range is so low that the amount of the radiation heat can be neglected for purpose of cell thermal analysis work.

\subsection{Development of Thermal Model of Li-Ion Cell}

\subsubsection{Heat Generation Mechanism}

For the thermal modelling of the Li-Ion cell, both reversible and irreversible heats were considered. The irreversible heat consists of the Joule effect due to the internal resistances in the cell. Based on the electric model approach of the cell as shown in figure 6 , two main resistances are considered in this work: the polarization resistance $R_{1}$ and the Ohmic resistance $R_{i}$. The equation for the heat generated at a central point in the cell is:

$$
\mathrm{Q}_{\text {heat,Joule }}=\mathrm{R}_{\mathrm{i}} \mathrm{I}^{2}+\mathrm{R}_{1} \mathrm{I}_{1}^{2}
$$

The reversible heat generation mechanism consists of the entropy effect can be negative or positive depending on charging or discharging process.

Based on measurement of a 63 Ah cell the initial model parameters have been optimized with Recursive Least Square (RLS) method. The obtained results show that the estimated values of internal resistance, capacity, OCV and others provide valuable information about cell internal/external temperature and terminal voltages even if the physical sensors become faulty and go out of operation for any reason.

The heat generated by the entropy variation depends strongly on the OCV and therefore the SOC as shown in figure 17 for the mentioned cell. The equation for the reversible heat can be written as equation 6 :

$$
Q_{\text {heat }, r e v}=-T \Delta S \frac{I}{n F}
$$

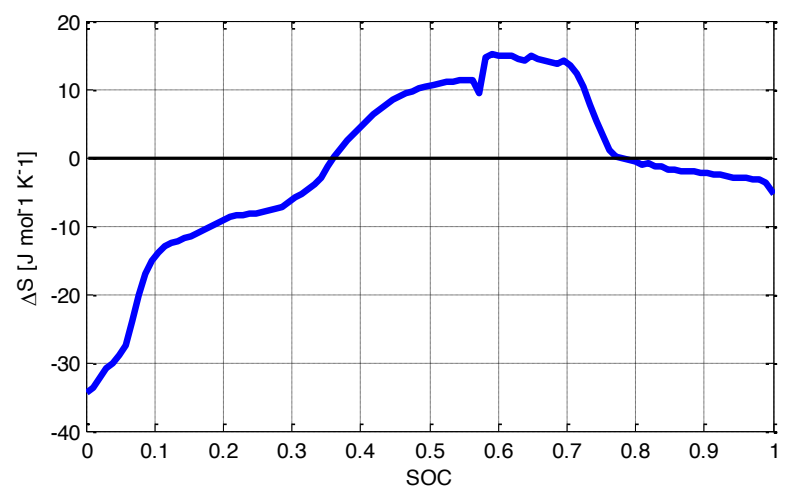

Figure 17: Calculated entropy vs SOC 
In equation $5, \mathrm{~F}$ is the Faraday constant.

Thus, the total heat generated can be written as summation of Equation 4 and 5:

$Q_{\text {heat,total }}=Q_{\text {heat }, \text { Joule }}+Q_{\text {heat,rev }}$

\subsubsection{Heat Transfer Effects}

1) Conductive Heat Transfer

$Q_{\text {cond }}=\frac{k A}{d}\left(T_{i}-T_{\text {cell }}\right)$

Where $\mathbf{k}$ is the thermal conductivity, $\mathbf{A}$ is the cross section area of the cell, $\mathbf{d}$ is the cell thickness, $\mathbf{T}_{\mathbf{i}}$ is the cell estimated internal temperature and $\boldsymbol{T}_{\text {cell }}$ is the temperature on the surface of the cell.

2) Convective Heat Transfer

$Q_{\text {conv }}=h A\left(T_{\text {cell }}-T_{a m b}\right)$

$\mathbf{h}$ is the Heat transfer coefficient and $\mathbf{T}_{\mathbf{a m b}}$ is the ambient temperature.

\subsection{Mathematical Formulation and Parameterization of the Cell Thermal Model}

In the formula presented in Equation $9, \mathbf{c}_{\mathbf{p}}$ is the specific heat capacity, $\mathbf{m}$ is mass of the cell, initial value of each parameter is calculated in offline mode and presented in table 1 .

$$
\mathrm{c}_{\mathrm{p}} \mathrm{m} \frac{\partial \mathrm{T}_{\mathrm{i}}}{\partial \mathrm{t}}=\mathrm{Q}_{\text {heat,total }}-\frac{\mathrm{kA}}{\mathrm{d}}\left(\mathrm{T}_{\mathrm{i}}-\mathrm{T}_{\text {cell }}\right)
$$

We consider that the amount of heat flow absorbed by conduction effect is the same as the one absorbed by convection. We can write the following equation:

$\mathrm{Q}_{\text {cond }}=\mathrm{Q}_{\text {conv }}$

From this equation, we can derive the relation between $\mathrm{T}_{\mathrm{amb}}$ and $\mathrm{T}_{\mathrm{i}}$,

$\mathrm{T}_{\text {cell }}=\frac{\mathrm{k} \cdot \mathrm{T}_{\mathrm{i}}+\mathrm{hd} \cdot \mathrm{T}_{\mathrm{amb}}}{\mathrm{hd}+\mathrm{k}}$
Parameters $\mathbf{k}, \mathbf{h}$ and $\mathbf{c}_{\mathbf{p}}$ will be identified by training the data with real measurements. All necessary heat coefficients are brought in table 2 . Figure 18 demonstrates the comparison between cell measured temperature and cell modelled temperature as a result of the input current profile shown in Figure 16 (a).

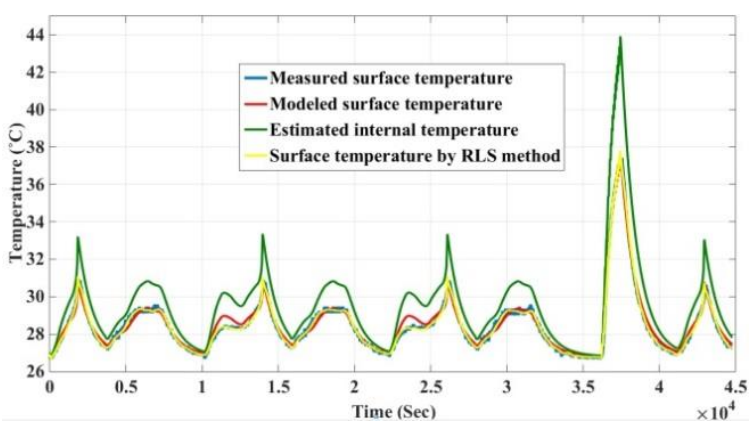

Figure 18: Measured and estimated temperature of the cell as the result of test profile shown is figure 16 (a)

Table 1: Physical Cell Parameters

\begin{tabular}{|c|c|c|c|}
\hline Parameter & mass & $\begin{array}{c}\text { cross } \\
\text { section area }\end{array}$ & thickness \\
\hline Sign & $\mathrm{M}(\mathrm{kg})$ & $\mathrm{A}\left(\mathrm{m}^{2}\right)$ & $\mathrm{d}(\mathrm{m})$ \\
\hline Value & 1.52 & $0.262 * 0.257$ & 0.0053 \\
\hline
\end{tabular}

Table 1: Calculated heat coefficients of the cell

\begin{tabular}{|c|c|c|c|}
\hline Parameter & $\begin{array}{c}\text { Thermal } \\
\text { conductivity }\end{array}$ & $\begin{array}{c}\text { Heat } \\
\text { transfer } \\
\text { coefficient }\end{array}$ & $\begin{array}{c}\text { Specific } \\
\text { heat } \\
\text { capacity }\end{array}$ \\
\hline Sign & $\mathrm{k}$ & $\mathrm{h}$ & $\mathrm{c}_{\mathrm{p}}$ \\
$\left(\frac{\mathrm{W}}{\mathrm{mK}}\right)$ & $\left(\frac{\mathrm{W}}{\mathrm{m}^{2} \mathrm{~K}}\right)$ & $(\mathrm{J} / \mathrm{kg} / \mathrm{K})$ \\
\hline Value & 0.3627 & 38.9532 & 1.1044 \\
\hline
\end{tabular}




\section{Temperature Distribution in the Cell}

The ECM assumes the same temperature for the whole cell. In large format high power Li-Ion cells, like the one investigated, the temperature is likely to vary locally. At the same time it is difficult to measure the spatial temperature distribution within the cell [10]. However multidimensional finite element method (FEM) models provide valuable assumptions of the temperature gradients and local heat generation or dissipation inside a single battery cell or cell layer. FEM models are not suitable for BMS application, as they do not provide real time data. Nevertheless they facilitate a better understanding of the physiochemical processes in the battery cell as these are explained in greater detail.

The electrochemical-thermal COMSOL models employ the Porous Electrode Theory and the Concentrated Solution Theory introduced by Newman and Tideman [11] and Doyle et al. [12]. The cell behaviour is characterized by its reaction kinetics, charge transfer and its internal material transport including diffusion reactions. For the single layer model these reactions are coupled to the energy balance to simulate the thermal behaviour [13]. For a lumped 2D analysis of the entire cell stack, the single layer properties are averaged over the entire layer to create a heat rate estimation at different boundary temperatures and times of the cycling process. This estimation is then fed into a purely thermal cell stack model described by the unsteady heat conduction equation.

$\rho c_{p} \dot{T}=-k \Delta \nabla^{2} T+Q$

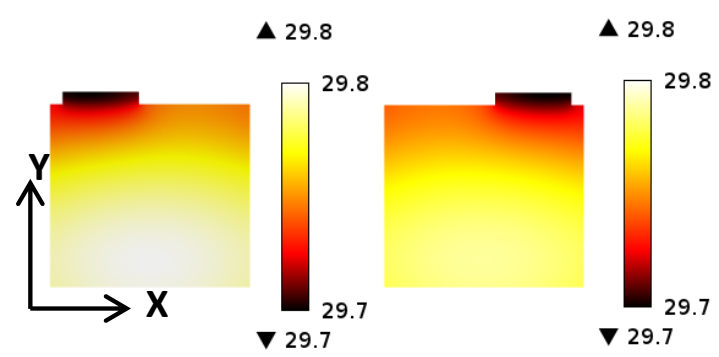

Figure 19: Simulated temperature distribution in a fully discharged $63 \mathrm{Ah}$ high power Li-Ion pouch cell in the middle plane of the cell (xy-plane) at the end of $1 \mathrm{C}$ discharge cycle
Simulations show temperature gradients do exist within the large format high power Li-Ion pouch cell for EV applications. However they remain rather small. Figure 19 shows the spatial temperature distribution in the 63 Ah high power Li-Ion pouch cell along the surface of the cell stack within the symmetry plane (xy-plane) at the end of $1 \mathrm{C}$ discharge cycle. The temperature gradient is about $-0.2^{\circ} \mathrm{C}$. At $5 \mathrm{C}$ constant discharge, the in-plane difference is merely higher at about $0.9{ }^{\circ} \mathrm{C}$ for a fully charge cell (figure 20). This applies to a simple constant charge/discharge of a single cell with a huge surface area and thus high heat dissipation only. This temperature gradient is negligible at normal discharge rate, except in case of higher discharge current, where the temperature gradient is more than $1^{\circ} \mathrm{C}$. For higher C-Rates pulses (8C and above) or in a tightly packed battery module, where the cells will heat each other up, this will not the case and the temperature rise and temperature in plane difference is higher. Not to talk about abuse conditions, this causes local heating within the battery cell.

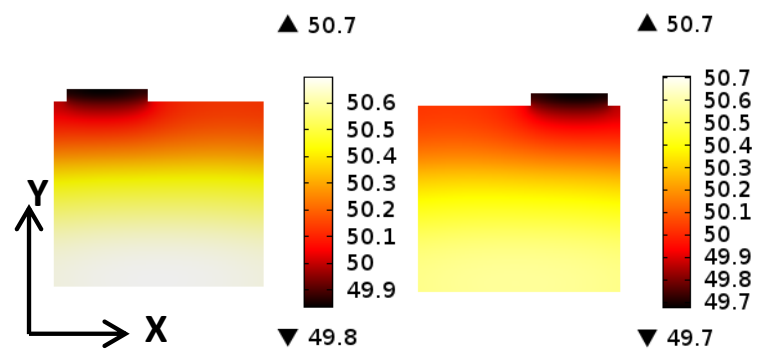

Figure 20: Simulated temperature distribution in a fully charged 63 Ah high power Li-Ion pouch cell in the middle plane of the cell (xy-plane) at the end of $5 \mathrm{C}$ discharge cycle

Yet, during standard operation, the local temperature differences remain negligible and are therefore not considered in the online ECM models for BMS application.

Both COMSOL model and SIMULINK model were evaluated with temperature sensor data. Sensor measurement and SIMULINK model are shown in figure 18 and COMSOL model is shown in figure 19 for $1 \mathrm{C}$ discharge for both anode and cathode sides. The test profile to generate input for both simulation models is shown in figure 16(a). After about 10,000 seconds of the test, during the discharge part of the cell, there is an increase in the cell temperature. At the end of the discharge after about 4,000 seconds both models show a temperature rise from room temperature at $26{ }^{\circ} \mathrm{C}$ to about $30{ }^{\circ} \mathrm{C}$. This one to one comparison between 
models and evaluation with temperature sensor data at various test profiles such as drive cycles or pulse sequences etc. during charge/discharge we successfully evaluated and the algorithms were implemented in a low cost target microcontroller.

\section{Conclusion}

This paper presents a novel coupled electrical and thermal model for large format high power Li-ion cell in terms of interaction between models and considering temperature effect for tropical weather condition for countries like Singapore and also cycling ageing of the cell. Three new 63 Ah large pouch cells were selected for each test matrix to have statistically reliable data and also generating comprehensive and enough amounts of reliable reference data for comparison purposes between measurements and Simulations. The electrical model is known as ECM model and the parameterization technique is explained in detail. ECM model was evaluated real time by HIL system and was implementable on target microcontroller and BMS system. Thermal model uses the inputs of ECM to generate simulated temperature of the cell and feedbacks the simulation data into the ECM model to complete the interaction feedback loop. As cycling ageing and temperature effects were considered and implemented in both ECM and thermal model of the cell, this coupled model is suitable to be up-scaled into battery pack model and be used in BMS. Moreover the cell surface and internal temperature of the cell were estimated by the thermal model in MATLAB and COMSOL. For evaluation of the estimated internal temperature, a cell sandwich experiment is proposed, where the temperature sensor is placed between two pouch cells surfaces.

\section{Acknowledgment}

This work has been done in the framework of CREATE research program funded by the Singapore National Research Foundation (NRF).

\section{References}

H. He, R. Xiong, H. Guo, and S. Li,

"Comparison study on the battery models used for the energy management of batteries in electric vehicles," Energy Convers. Manag., vol. 64, pp. 113-121, Dec. 2012.

[2] W. Waag, "“ Adaptive algorithms for monitoring of lithium-ion batteries in electric vehicles ," 2014.

[3] G. G. Botte, R. E. White, and Z. Zhang, "Thermal stability of LiPF $6 \pm$ EC : EMC electrolyte for lithium ion batteries," vol. 98, pp. 570-575, 2001.

[4] D. O. E. Id-, "PNGV Battery Test Manual," no. February, 2001.

[5] W. Waag, C. Fleischer, and D. U. Sauer, "On-line estimation of lithium-ion battery impedance parameters using a novel variedparameters approach," J. Power Sources, vol. 237, pp. 260-269, Sep. 2013.

[6] R. Arunachala, K. Makinejad, S. Athlekar, A. Jossen, and J. Garche, "Cycle life characterisation of large format lithium-ion cells," in 2013 World Electric Vehicle Symposium and Exhibition (EVS27), 2013, pp. 1-9.

[7] M. W. Verbrugge and P. Liu, "Electrochemical characterization of highpower lithium ion batteries using triangular voltage and current excitation sources," $J$. Power Sources, vol. 174, no. 1, pp. 2-8, Nov. 2007.

[8] N. Williard, W. He, M. Osterman, and M. Pecht, "Comparative Analysis of Features for Determining State of Health in Lithium-Ion Batteries," 2013.

[9] H. Song, Z. Cao, X. Chen, H. Lu, M. Jia, Z. Zhang, Y. Lai, J. Li, and Y. Liu, "Capacity fade of LiFePO4/graphite cell at elevated temperature," J. Solid State Electrochem., vol. 17, no. 3, pp. 599-605, Oct. 2012.

[10] N. Martiny, A. Rheinfeld, J. Geder, Y. Wang, W. Kraus, and A. Jossen, "Development of an All Kapton-Based Thin-Film Thermocouple Matrix for In Situ Temperature Measurement in a Lithium Ion Pouch Cell," vol. 14, no. 10, pp. 3377-3384, 2014. 
[11] J. Newman and W. Tiedemann, "Porouselectrode theory with battery applications," AIChE J., vol. 21, no. 1, pp. 25-41, Jan. 1975.

[12] M. Doyle, "Modeling of Galvanostatic Charge and Discharge of the Lithium/Polymer/Insertion Cell," $J$. Electrochem. Soc., vol. 140, no. 6, p. 1526, 1993.

[13] D. Bernardi, “A General Energy Balance for Battery Systems," J. Electrochem. Soc., vol. 132 , no. 1 , p. 5,1985 .

\section{Authors}

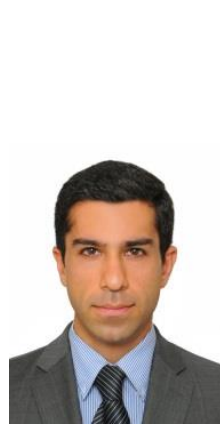

Kamyar Makinejad earned his M.Sc. degree from Leibniz University of Hanover, Germany in 2011. He is currently working as Research Associate in TUM CREATE, Singapore and pursuing his $\mathrm{PhD}$ with the Technical University of Munich, Germany. His research interest is battery diagnostics, modelling, state determination and BMS in EV applications.

Raghavendra Arunachala earned his B.Eng. in electrical and electronic engineering from Visveswaraya

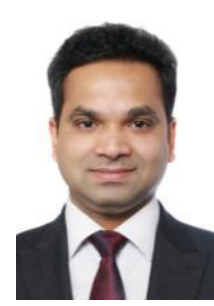
Technological University, India. He completed his M.Sc from Rheinische Westfälische Technische Hochschule (RWTH) Aachen University, Germany in 2011. He is currently a doctoral student in TUM CREATE, Singapore, working in the area of electrical energy storage systems

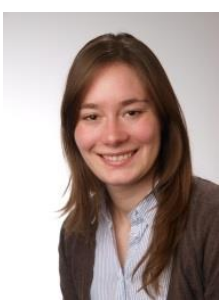

Sabine Arnold studied Electrical Engineering and Information Technology at Karlsruhe Institute for Technology (KIT), Germany. Her research within TUM CREATE focuses on battery modelling on cell level. Here, she is especially interested in 3D, multiphysic models, which allow safety simulation.

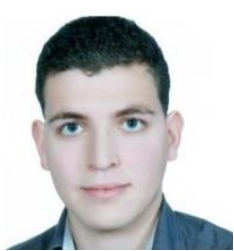

Hassen Ennifar earned his B.Sc. in electrical engineering and information from Nanyang Technological University, currently his is a master student at Technische Universität München, German.

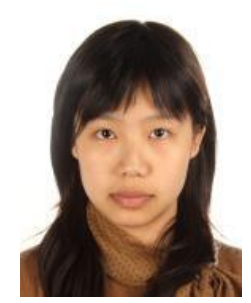

Han Zhou obtained her bachelor degree in Material Science and Engineering from Nanjing University, China, and master degree in Material Science from Université de Picardie Jules Verne, Amiens, France. Currently she is working in TUM CREATE on thermal management of battery pack.

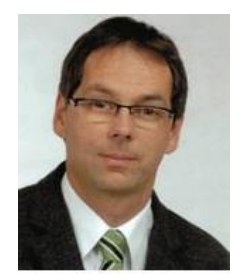

Andreas Jossen earned his doctorate, dealing with "Management of photovoltaic plants using energy storage systems" at University of Stuttgart. From 1994 he was group leader for different battery related topics with ZSW, Ulm. Since 2010 he is full professor at the Institute for Electrical Energy Storage Technology, TUM. Prof. Jossen is responsible for Research Project 2 (RP2) and RP6 at TUM CREATE, Singapore.

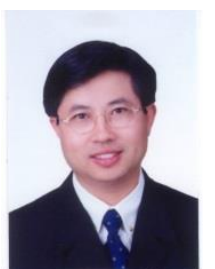

Changyun Wen received his B.Eng. from Xi'an Jiaotong University, China in 1983 and Ph.D. from the University of Newcastle, Australia in 1990. He joined the School of Electrical and Electronic Engineering at Nanyang Technological University (NTU) as a lecturer. He was promoted to Full Professor through the first promotion and Tenure Review Exercise at NTU in 2008. 